\title{
The Effects of Preoperative Oral Carbohydrate on Frequency of $T$ and NK Cells in Patients with Cervical Cancer Treated Using Neoadjuvant Chemotherapy and Surgery: A Prospective Cohort Study
}

\author{
Fuqing Zhang, ${ }^{1}$ Mengxia Yao, ${ }^{1}$ Zhiping Lin, ${ }^{1}$ Yili Chen, ${ }^{1}$ Hui Jiang, ${ }^{1}$ Meina Zeng, \\ and Wenhua Chen $\Phi^{2}$ \\ ${ }^{1}$ Department of Anesthesiology, Fujian Cancer Hospital \& Fujian Medical University Cancer Hospital, China \\ ${ }^{2}$ Department of Anesthesiology, Fujian Medical University Union Hospital, China \\ Correspondence should be addressed to Wenhua Chen; whc6202@163.com
}

Received 26 November 2019; Revised 27 February 2020; Accepted 2 March 2020; Published 17 March 2020

Academic Editor: Harry W. Schroeder

Copyright @ 2020 Fuqing Zhang et al. This is an open access article distributed under the Creative Commons Attribution License, which permits unrestricted use, distribution, and reproduction in any medium, provided the original work is properly cited.

Background. Immune dysfunction can occur after neoadjuvant chemotherapy (NAC) and surgery for cancer. We investigated whether preoperative oral carbohydrate affected the postoperative percentages of $\mathrm{T}$ cells $\left(\mathrm{CD} 4^{+}\right.$and $\left.\mathrm{CD} 8^{+}\right)$and natural killer (NK) cells in patients with cervical cancer treated with NAC and surgery. Methods. This prospective cohort study enrolled consecutive patients with cervical cancer treated by radical hysterectomy with PLND at the Gynecologic Oncology Department of Fujian Provincial Cancer Hospital (China) between January 2018 and December 2018. Patients were divided into three groups according to the treatment method: NAC (two cycles, surgery 1 month later), NAC+CHO (chemotherapy and surgical methods same as with the NAC group but with $300 \mathrm{~mL}$ of oral carbohydrate administered $2 \mathrm{~h}$ before surgery), and non-NAC (surgery alone). Percentages of $\mathrm{NK}, \mathrm{CD}^{+}, \mathrm{CD}^{+}$, and $\mathrm{CD}^{+}$cells were evaluated by flow cytometry the day after the first admission, just before surgery, immediately after tracheal tube removal, and the day after surgery. This trial is registered with NCT03872635 at clinicaltrials.com. Results. The final analysis included 77 patients (non-NAC group, $n=26$; NAC group, $n=25$; and NAC-CHO group, $n=26)$. Baseline characteristics and preoperative $\mathrm{NK}, \mathrm{CD}^{+}, \mathrm{CD}^{+}$, and $\mathrm{CD}^{+}$cell percentages were similar between groups. Postoperatively, all groups exhibited reductions in $\mathrm{NK}, \mathrm{CD}^{+}$, and $\mathrm{CD} 4^{+}$cell percentages and increases in $\mathrm{CD}^{+}$cell percentages (all $\mathrm{P}<0.05$ ). The changes in $\mathrm{NK}, \mathrm{CD}^{+}, \mathrm{CD}^{+}$, and $\mathrm{CD}^{+}$cell percentages were attenuated in the NAC-CHO group ( $P<0.05$ vs. both other groups). Conclusion. Preoperative oral carbohydrate can improve the postoperative populations of NK and T cells after the treatment of cervical cancer by NAC and surgery.

\section{Introduction}

Cervical cancer is the fourth most common malignancy in females [1]. In 2015, the incidence rate of cervical cancer in China was $9.89 \%$ [2]. Therapies for cervical cancer include radical hysterectomy, chemotherapy, radiotherapy, and concurrent chemoradiotherapy [3]. However, the recurrence rate is $10 \%-50 \%$, and the 5 -year survival is around $65 \%$, although this varies with the disease stage [4].

Radical hysterectomy $(\mathrm{RH})$ with pelvic lymph node dissection (PLND) is one treatment option recommended by the National Comprehensive Cancer Network (NCCN) guidelines for stage IB and IIA cervical cancer [5]. Preoperative neoadjuvant chemotherapy (NAC) can reduce tumor size and facilitate surgical resection [6]. NAC results in a response in $53-79 \%$ of patients with cervical cancer [7-9], and its reported clinical benefits include improved surgical feasibility, reduced need for postoperative chemoradiation therapy, and longer progression-free survival and overall survival $[10,11]$.

The suppression of normal immune responses by cancer cells is an important mechanism contributing to tumor 
growth and proliferation [12], and there is increasing interest in the roles of lymphocytes such as $\mathrm{CD} 4^{+}$cells (helper T cells and regulatory $\mathrm{T}$ cells), $\mathrm{CD}^{+}$cells (cytotoxic $\mathrm{T}$ cells), and natural killer (NK) cells. Helper T cells facilitate other lymphocytes and activate macrophages and cytotoxic $\mathrm{T}$ cells. Regulatory $\mathrm{T}$ cells inhibit $\mathrm{T}$ cell-mediated immunity and are involved in immunologic tolerance. Cytotoxic $\mathrm{T}$ cells and NK cells can target and destroy tumor cells. Previous studies of patients with cervical cancer have described alterations in $\mathrm{T}$ cell and NK cell populations [13], suppression of $\mathrm{NK}$ cell activity by $\mathrm{CD}^{+}$regulatory $\mathrm{T}$ cells [14], and reduced numbers of $\mathrm{CD}^{+}{ }^{+} \mathrm{T}$ cells and NK cells in the neoplastic cervix [15]. Importantly, $\mathrm{RH}$ for cervical cancer has been reported to decrease $\mathrm{CD} 4^{+} \mathrm{T}$ cell and $\mathrm{NK}$ cell numbers and the $\mathrm{CD}^{+} / \mathrm{CD}^{+}$ratio [16], indicating that surgery can cause immune dysregulation. Although NAC for cervical cancer appears not to suppress lymphocyte numbers, lower levels of $\mathrm{CD}^{+} \mathrm{T}$ cells, $\mathrm{CD} 8^{+} \mathrm{T}$ cells, and NK cells are associated with a poorer response to chemotherapy $[17,18]$. Additionally, polychemotherapy may inhibit NK cell activity without reducing NK cell numbers [19].

Reduced calorie intake has been shown to decrease CD $4^{+}$ and $\mathrm{CD}^{+}$cell numbers in patients with rheumatoid arthritis [20], suggesting that preoperative fasting may contribute to the decline in lymphocyte numbers after surgery. There is increasing interest in the potential benefits of administering high-carbohydrate drinks before surgery. Oral carbohydrate intake rather than fasting before surgery can suppress postoperative metabolic disturbances such as insulin resistance, improve postoperative cardiac function, enhance postoperative patient comfort, and shorten hospital stay without increasing the incidence of complications [21-28]. Additionally, the preoperative oral carbohydrate may help to ameliorate the decline in $\mathrm{T}$ lymphocytes and NK cells following surgery [23].

We hypothesized a preoperative oral carbohydrate drink would attenuate postoperative immune system disturbances in patients treated for cervical cancer using NAC and surgery. The aim of this study was to investigate whether preoperative oral carbohydrate would attenuate postoperative changes in $\mathrm{CD}^{+} \mathrm{T}$ cell, $\mathrm{CD} 8^{+} \mathrm{T}$ cell, and NK cell numbers in patients with cervical cancer undergoing $\mathrm{RH}$ and PLND after NAC.

\section{Material and Methods}

2.1. Study Design and Participants. This prospective cohort study enrolled consecutive patients with cervical cancer treated by RH and PLND at the Gynecologic Oncology Department, Fujian Provincial Cancer Hospital, Fujian Medical University, Fuzhou, Fujian, China, between January 2018 and December 2018. The inclusion criteria were as follows: (1) female aged 18-60 years; (2) American Society of Anesthesiologists (ASA) level I or II; (3) cervical squamous cell carcinoma confirmed by cervical biopsy and pathology; (4) no distant metastasis observed on computed tomography (CT); (5) CT, magnetic resonance imaging (MRI), positron emission tomography (PE), and/or B-ultrasound and gynecologic examinations indicated stage IB1 or IIA1 disease with a cervical tumor mass of $2-4 \mathrm{~cm}$ (non-NAC group) or stage IB2 or IIA2 disease with a cervical tumor mass $>4 \mathrm{~cm}$ (NAC group); (6) the planned surgery was laparoscopically assisted $\mathrm{RH}$ with bilateral salpingo-oophorectomy and PLND; and (7) the planned chemotherapy regimen (NAC groups) was two courses of paclitaxel and cisplatin, with an interval of 4 weeks between the second course and surgery. The exclusion criteria were as follows: (1) previous chemotherapy (for patients in the NAC groups); (2) dysfunction of gastric emptying; (3) diabetes mellitus or impaired glucose tolerance; (4) body mass index $(\mathrm{BMI})>32$ or $<18.5 \mathrm{~kg} / \mathrm{m}^{2}$; (5) multiple cervical tumors; (6) tumor recurrence; and (7) functional disorders of the respiratory, circulatory, immune, nervous, or urinary system or any other organs. Additionally, patients were excluded from the final analysis if they received a blood transfusion or nutritional support within the 2 weeks before surgery or were given a carbohydrate-containing solution, blood transfusion, glucocorticoid, or ephedrine (to treat hypotension) during surgery.

This study was performed in accordance with the principles of the Declaration of Helsinki and approved by the ethics committee of Fujian Medical University (KT2008-003-01). All patients provided informed written consent before enrolment. This study is registered at clinicaltrials.com (NCT03872635).

2.2. Patient Grouping. Patients were divided into three groups using a computer-generated random number table: the NAC group (stage IB2/IIA2 disease and a cervical tumor mass $>4 \mathrm{~cm}$ ) treated using NAC followed by RH/PLND; the $\mathrm{NAC}+\mathrm{CHO}$ group (stage IB2/IIA2 disease and a cervical tumor mass $>4 \mathrm{~cm}$ ) treated using NAC, preoperative oral carbohydrate, and then RH/PLND; and the non-NAC group (stage IB1/IIA1 disease and a cervical tumor mass of 2-4 cm) treated by RH/PLND alone. Neither the participants nor the investigators were blinded to the intervention.

2.3. NAC. The patients in the NAC and NAC+CHO groups received two cycles of NAC. The 3-week chemotherapy regimen consisted of paclitaxel $\left(135-175 \mathrm{mg} / \mathrm{m}^{2}\right)$ and cisplatin $\left(75 \mathrm{mg} / \mathrm{m}^{2}\right)$. The interval between the end of the second cycle and surgery was 4 weeks.

2.4. Preoperative Preparation. All patients undertook preoperative bowel preparation. Patients in the non-NAC and NAC groups underwent an 8-hour preoperative fast for solids and a 4-hour preoperative fast for fluids. Patients in the $\mathrm{NAC}+\mathrm{CHO}$ group underwent an 8-hour preoperative fast for solids, consumed $300 \mathrm{~mL}$ of an oral carbohydrate drink 2 hours before surgery, and then underwent a 2-hour preoperative fast for fluids. The main components of the carbohydrate drink (Shuneng multifunctional beverage, Yichang Renfu Pharmaceutical Co., Ltd.; batch number: S20160629) were carbohydrate, sodium, vitamin B1, vitamin B6, vitamin B12, zinc, and taurine.

2.5. Surgery. All operations started between 09:00 and 09:30 and were conducted by the same team of anesthesiologists and surgeons. Anesthesia was induced using midazolam (0.5 mg/kg; Jiangsu Enhua Pharmaceutical Co., Ltd.), fentanyl citrate $(4 \mu \mathrm{g} / \mathrm{kg}$; Yichang Renfu Pharmaceutical Co., 
Ltd.), propofol (2 mg/kg; B. Braun Melsungen AG), and rocuronium bromide $(8 \mathrm{mg} / \mathrm{kg}$; Huabei Pharmaceutical Co., Ltd.). After tracheal intubation, general anesthesia was maintained using inhaled sevoflurane (1-2\%; Shanghai Hengrui Pharmaceutical Co., Ltd.), propofol $(2-5 \mathrm{mg} / \mathrm{kg} / \mathrm{h})$, remifentanil hydrochloride $(0.05-0.15 \mu \mathrm{g} / \mathrm{kg} / \mathrm{min}$; Yichang Renfu Pharmaceutical Co., Ltd.), and cisatracuriumbesylate (2-3 $\mu \mathrm{g} / \mathrm{kg} / \mathrm{min}$; Zhejiang Xianju Pharmaceutical Co., Ltd.) with a tidal volume of $6-8 \mathrm{~mL} / \mathrm{kg}$, a respiratory frequency of 10-12 times/min, and an end-expiratory carbon dioxide partial pressure of $35-45 \mathrm{mmHg}$. Intraoperative hypotension (blood pressure fall $>30 \%$ of the baseline value), hypertension (blood pressure rise $>30 \%$ of the baseline value), bradycardia, and tachycardia were managed by an intravenous injection of $10 \mathrm{mg}$ ephedrine, $5 \mathrm{mg}$ urapidil, $0.5 \mathrm{mg}$ atropine, and $10 \mathrm{mg}$ esmolol, respectively. Patients administered with ephedrine intraoperatively were excluded from the final analysis.

All patients underwent laparoscopically assisted $\mathrm{RH}$ with bilateral salpingo-oophorectomy and PLND. Postoperatively, the patients were fasted for 24 hours and given amino acids, fatty acids, and glucose for parenteral nutritional support. Postoperative analgesia involved the intravenous infusion of a solution $(100 \mathrm{~mL})$ containing $150 \mu \mathrm{g}$ sufentanil citrate (Yichang Renfu Pharmaceutical Co., Ltd.), $150 \mathrm{mg}$ flurbiprofen ester (Beijing Tide Pharmaceutical Co., Ltd.), and $15 \mathrm{mg}$ tropisetron ( $2 \mathrm{~mL} /$ hour fixed flow).

2.6. Detection of Lymphocyte Subsets. For the three groups of patients, we compared the percentages of peripheral NK cells and $\mathrm{T}$ cell subsets, including CD3+\% (percentage of total $\mathrm{T}$ cells), CD4+\% (percentage of helper T cells), CD8+\% (percentage of inhibitory/cytotoxic cells), and CD4+/CD8+ ratio at 6:00 am the next day after the first hospital admission, at 6:00 am on the day of surgery, at the time of extubation, and at 6:00 am the next day after surgery. The blood sample was centrifuged at $4^{\circ} \mathrm{C}, 3000 \mathrm{rpm}$, for $10 \mathrm{~min}$, to separate the red blood cells, buffy coat, and plasma. Then, the plasma was separated, and the buffy coat was entirely taken for flow cytometry. T lymphocyte subsets and NK cells were evaluated in peripheral venous blood using flow cytometry (BD FACSCalibur flow cytometer; BD Biosciences). The forward scatter (FSC) and side scatter (SSC) of the excitation light reflected the cell size and internal structure, respectively. The distributions and quantities of lymphocytes expressing CD3 (mature T cells), CD4 (helper and regulatory T cells), and CD8 (cytotoxic T cells) were evaluated using specific antibodies (BD Lymphocyte Subset Detection Reagent and BD NK Cell Detection Reagents) and immunofluorescence. The CellQuest software was used for acquiring and analyzing data.

2.7. Clinical Data. The following data were recorded: age, BMI, operative time, intraoperative blood loss, and infusion volume. The percentages of NK cells, $\mathrm{CD}^{+}$cells, $\mathrm{CD} 4^{+}$cells, and $\mathrm{CD}^{+}$cells $(\mathrm{NK} \%, \mathrm{CD} 3 \%, \mathrm{CD} 4 \%$, and $\mathrm{CD} 8 \%$, respectively) and the ratio of $\mathrm{CD}^{+}$cells to $\mathrm{CD}^{+}$cells (CD4/CD 8 ratio) in peripheral venous blood were determined at four time points: the day after the first admission; 06:00 on the day of surgery; immediately after removal of the tracheal tube; and 06:00 on the day after surgery. The main outcome measures were postoperative NK\%, CD3\%, CD4\%, CD8\%, and $\mathrm{CD} 4 / \mathrm{CD} 8$ ratio.

2.8. Sample Size Calculation. The patients in this study had been recruited in a clinical trial (NCT03872635), assessing the effects of preoperative oral carbohydrate on insulin resistance and immune cell levels after surgery. Sample size calculation in the original trial was based on a preliminary assessment of blood glucose levels at 24 hours after surgery; the values were $6.00 \pm 3.40 \mathrm{mmol} / \mathrm{L}$ in patients treated with surgery only, $5.07 \pm 5.25 \mathrm{mmol} / \mathrm{L}$ in patients treated with $\mathrm{NAC}$ and surgery, and $7.32 \pm 2.35 \mathrm{mmol} / \mathrm{L} \quad(n=10$ per group) in patients treated with NAC, preoperative oral carbohydrate, and surgery. The minimum sample size was calculated using the following formula:

$$
n=\varphi^{2} \frac{\left(\sum \mathrm{si}^{2} / k\right)}{\sum(\mathrm{xi}-\mathrm{xmean})^{2} /(k-1)}
$$

The parameters are as follows: (1) $\alpha=0.05$; (2) $\beta=0.10$; (3) $k$ is number of groups; (4) $\varphi$ is, in this example, where $k=3$, degrees of freedom of $V 1=k-1=2$, degrees of freedom of $V 2=N-1, N$ was unknown and could take a maximum of $\infty$, and using a look-up table, $\varphi \alpha, \beta, k-1, \infty=2.52$; (5) $\mathrm{xi}$ and si were, respectively, the mean of the $i$ group $(\mathrm{x} 1=6.00, \mathrm{x} 2=\cdots)$ and the standard deviation of the $i$ group $(\mathrm{s} 1=3.40, \mathrm{~s} 2=\cdots)$; and (6) determination of xmean is xmean $=(x 1+x 2+x 3) / k$.

The minimum sample size was calculated to be 74 and was expanded by $10 \%$ to take into account the loss to follow-up. Therefore, 81 patients (27 per group) were enrolled.

2.9. Statistical Analysis. The analysis was performed using SPSS 22.0 (IBM Corp., Armonk, NY, USA). Continuous data are expressed as the mean \pm standard deviation. Normally distributed continuous data were compared between using the independent samples $t$-test (two groups) and using one-way analysis of variance (ANOVA; three groups). Nonnormally distributed continuous data were compared between groups using the rank-sum test. Intragroup comparisons between different time points were made using repeated measures ANOVA. The chi-squared test was used for the comparisons of count data. $P<0.05$ was considered statistically significant.

\section{Results}

3.1. Baseline Clinical Characteristics of the Study Participants. Among the 81 patients initially enrolled in the study (27 in each group), one patient in the non-NAC group and two patients in the NAC group were excluded due to the intraoperative administration of ephedrine, and one patient in the $\mathrm{NAC}+\mathrm{CHO}$ group was excluded due to poor adherence to the study protocol (i.e., preoperative fasting and consumption of the oral carbohydrate drink). Therefore, the final analysis included 77 patients, with 26 in the non-NAC 


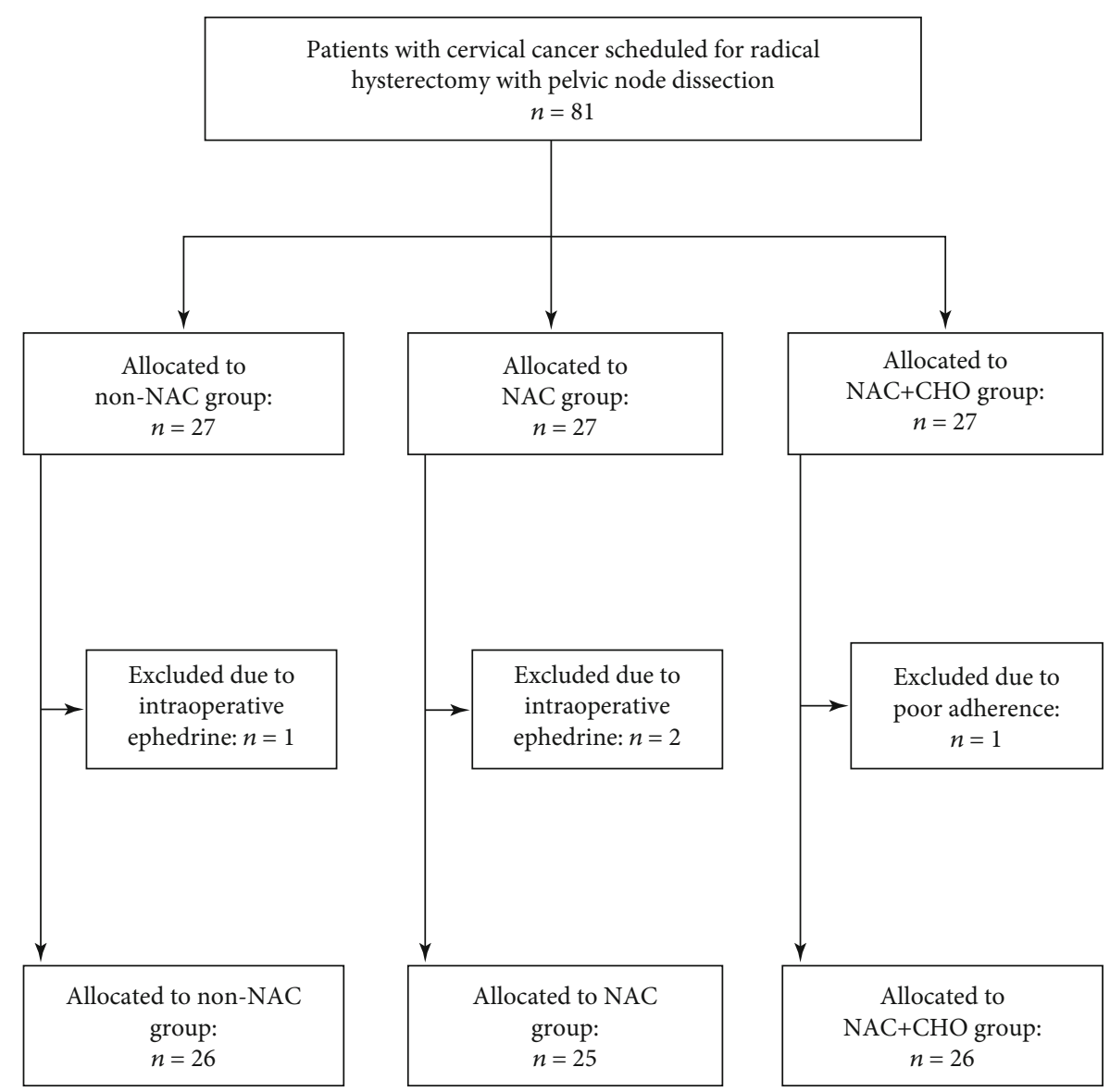

FIGURE 1: Flow chart showing patient enrollment in the study. CHO: carbohydrate; NAC: neoadjuvant chemotherapy.

TABLE 1: Baseline clinical characteristics of the study participants in the three groups.

\begin{tabular}{|c|c|c|c|c|c|}
\hline Characteristic & Non-NAC group $(n=26)$ & NAC group $(n=25)$ & $\mathrm{NAC}+\mathrm{CHO}$ group $(n=26)$ & $F$ & $P$ \\
\hline Age (years) & $52.92 \pm 7.56$ & $50.08 \pm 7.87$ & $53.65 \pm 8.40$ & 1.458 & 0.239 \\
\hline Body mass index $\left(\mathrm{kg} / \mathrm{m}^{2}\right)$ & $21.03 \pm 1.38$ & $20.99 \pm 0.99$ & $20.60 \pm 1.22$ & 1.011 & 0.369 \\
\hline Operative duration ( $\mathrm{min}$ ) & $180.50 \pm 19.19$ & $188.01 \pm 24.48$ & $187.74 \pm 17.89$ & 1.093 & 0.341 \\
\hline Blood loss (mL) & $211.7 \pm 30.09$ & $214.65 \pm 34.64$ & $221.54 \pm 36.23$ & 0.572 & 0.567 \\
\hline Infusion volume $(\mathrm{mL})$ & $1846.8 \pm 267.54$ & $1816.03 \pm 339.81$ & $1803.93 \pm 346.44$ & 0.124 & 0.883 \\
\hline
\end{tabular}

Data are presented as the mean \pm standard deviation.

group, 25 in the NAC group, and 26 in the $\mathrm{NAC}+\mathrm{CHO}$ group (Figure 1).

The baseline characteristics of the patients in each group are presented in Table 1. There were no significant differences between groups in patient age, BMI, operative time, intraoperative blood loss, and infusion volume (Table 1).

3.2. NK\% in Peripheral Venous Blood. There were no significant differences between groups in $\mathrm{NK} \%$ in peripheral venous blood on the day after the first admission and preoperatively on the day of surgery (Table 2). When compared with the preoperative value, NK\% was significantly lower after tracheal tube removal and on the day after surgery in all three groups $(P<0.05$; Table 2$)$. Notably, when compared with the other two groups, the NAC+CHO group had significantly higher $\mathrm{NK} \%$ after tracheal tube removal and on the day after surgery $(P<0.05$; Table 2$)$. Indeed, NK\% on the day after surgery was similar to that on the day after the first admission in the $\mathrm{NAC}+\mathrm{CHO}$ group but significantly lower than that on the day after the first admission in the other two groups (Table 2).

3.3. CD3\% in Peripheral Venous Blood. CD3\% (the percentage of lymphocytes that were mature $\mathrm{T}$ cells) in peripheral venous blood was similar between the three groups on the day after the first admission and preoperatively on the day of surgery (Table 3). All three groups showed significant declines in CD3\% after surgery $(P<0.05$; Table 3$)$. However, $\mathrm{CD} 3 \%$ on the day after surgery was significantly higher in the $\mathrm{NAC}+\mathrm{CHO}$ group than in the NAC group or non-NAC group $(P<0.05$; Table 3$)$. 
TABLE 2: Percentage of natural killer (NK) cells in peripheral venous blood.

\begin{tabular}{lcccc}
\hline Time point & Non-NAC group & NAC group & NAC+CHO group & $F^{2}$ \\
\hline Day after first admission & $14.29 \pm 8.39$ & $13.38 \pm 6.42$ & $12.84 \pm 9.10$ & 0.213 \\
Before surgery & $15.11 \pm 3.23$ & $16.17 \pm 4.73^{1}$ & $17.42 \pm 3.65^{\mathrm{a}, 1}$ & 2.261 \\
After tracheal tube removal & $5.93 \pm 2.84^{1,2}$ & $7.05 \pm 4.52^{\mathrm{a}, 1,2}$ & $12.73 \pm 5.12^{\mathrm{a}, \mathrm{b}, 2}$ & 104.388 \\
Day after surgery & $4.91 \pm 1.48^{1,2}$ & $5.83 \pm 2.93^{\mathrm{a}, 1,2,3}$ & $12.24 \pm 5.71^{\mathrm{a}, \mathrm{b}, 2}$ & 233.649 \\
$F^{1}$ & 35.821 & 44.839 & 5.942 & 0.111 \\
$P^{1}$ & 0.000 & 0.000 & 0.011 \\
\hline${ }^{1} P<0.05$ vs. day after the first admission; ${ }^{2} P<0.05$ vs. before surgery; ${ }^{3} P<0.05$ vs. after tracheal tube removal; ${ }^{\mathrm{a}} P<0.05$ vs. the non-NAC group; ${ }^{\mathrm{b}} P<0.05$ vs. \\
the NAC group.
\end{tabular}

TABLE 3: Percentage of $\mathrm{CD}^{+}$cells (all T cells) in peripheral venous blood.

\begin{tabular}{lcccc}
\hline Time point & Non-NAC group & NAC group & NAC+CHO group & $F^{2}$ \\
\hline Day after first admission & $71.21 \pm 9.88$ & $72.63 \pm 7.38$ & $73.16 \pm 6.83$ & 0.398 \\
Before surgery & $71.56 \pm 11.70$ & $76.21 \pm 12.62$ & $79.35 \pm 17.46$ & 0.673 \\
After tracheal tube removal & $63.84 \pm 6.72^{1,2}$ & $66.78 \pm 13.34^{1,2}$ & $73.29 \pm 25.94^{1,2}$ & 0.148 \\
Day after surgery & $64.58 \pm 5.26^{1,2}$ & $69.42 \pm 14.08^{1,2}$ & $76.39 \pm 23.82^{\mathrm{a}, 1,2}$ & 2.036 \\
$F^{1}$ & 6.080 & 2.920 & 0.522 & 3.464 \\
$P^{1}$ & 0.001 & 0.048 & 0.608 & 0.036 \\
\hline
\end{tabular}

${ }^{1} P<0.05$ vs. day after the first admission; ${ }^{2} P<0.05$ vs. before surgery; ${ }^{3} P<0.05$ vs. after tracheal tube removal; ${ }^{\text {a }} P<0.05$ vs. the non-NAC group; ${ }^{\mathrm{b}} P<0.05$ vs. the NAC group.

TABle 4: Percentage of $\mathrm{CD}^{+}$cells (helper and regulatory T cells) in peripheral venous blood.

\begin{tabular}{|c|c|c|c|c|c|}
\hline Time point & Non-NAC group & NAC group & $\mathrm{NAC}+\mathrm{CHO}$ group & $F^{2}$ & $P^{2}$ \\
\hline Day after first admission & $41.53 \pm 6.96$ & $44.87 \pm 9.16$ & $43.0 \pm 10.25$ & 0.918 & 0.404 \\
\hline Before surgery & $42.00 \pm 7.66$ & $49.49 \pm 5.57^{\mathrm{a}, 1}$ & $49.43 \pm 2.84^{\mathrm{a}, 1}$ & 14.807 & 0.502 \\
\hline After tracheal tube removal & $35.71 \pm 3.98^{1,2}$ & $32.86 \pm 6.95^{1,2}$ & $39.87 \pm 7.74^{\mathrm{a}, \mathrm{b}, 2}$ & 7.913 & 0.001 \\
\hline Day after surgery & $32.00 \pm 3.97^{1,2,3}$ & $29.73 \pm 3.03^{1,2}$ & $37.99 \pm 5.15^{\mathrm{a}, \mathrm{b}, 1,2}$ & 27.544 & 0.000 \\
\hline$F^{1}$ & 14.776 & 51.708 & 50.979 & & \\
\hline$P^{1}$ & 0.000 & 0.000 & 0.000 & & \\
\hline
\end{tabular}

${ }^{1} P<0.05$ vs. day after the first admission; ${ }^{2} P<0.05$ vs. before surgery; ${ }^{3} P<0.05$ vs. after tracheal tube removal; ${ }^{\mathrm{a}} P<0.05$ vs. the non-NAC group; ${ }^{\mathrm{b}} P<0.05$ vs. the NAC group.

TABle 5: Percentage of $\mathrm{CD}^{+}$cells (cytotoxic T cells) in peripheral venous blood.

\begin{tabular}{lcccc}
\hline Time point & Non-NAC group & NAC group & NAC+CHO group & $F^{2}$ \\
\hline Day after first admission & $25.56 \pm 7.49$ & $24.01 \pm 6.58$ & $25.88 \pm 9.36$ & 0.418 \\
Before surgery & $24.75 \pm 3.42$ & $19.54 \pm 5.38^{\mathrm{a}, 1}$ & $18.51 \pm 5.59^{\mathrm{a}, 1}$ & 0.660 \\
After tracheal tube removal & $30.63 \pm 3.51^{1,2}$ & $25.97 \pm 7.49^{\mathrm{a}, 2}$ & $19.40 \pm 2.58^{\mathrm{a}, \mathrm{b}, 1}$ & 3.508 \\
Day after surgery & $32.92 \pm 3.91^{1,2}$ & $28.88 \pm 2.94^{\mathrm{a}, 2}$ & $22.20 \pm 6.69^{\mathrm{a}}$ & 40.000 \\
$F^{1}$ & 15.853 & 6.077 & 5.040 & 0.000 \\
$P^{1}$ & 0.000 & 0.000 & 0.008 & 0.000 \\
\hline
\end{tabular}

${ }^{1} P<0.05$ vs. day after the first admission; ${ }^{2} P<0.05$ vs. before surgery; ${ }^{3} P<0.05$ vs. after tracheal tube removal; ${ }^{\mathrm{a}} P<0.05$ vs. the non-NAC group; ${ }^{\mathrm{b}} P<0.05$ vs. the NAC group.

3.4. CD4\% in Peripheral Venous Blood. As shown in Table 4, $\mathrm{CD} 4 \%$ (the percentage of cells that were helper and regulatory $\mathrm{T}$ cells) in peripheral venous blood was not significantly different between groups at the preoperative time points, but all three groups exhibited a lower CD $4 \%$ after surgery $(P<0.05)$. Furthermore, CD4\% on the day after surgery was significantly higher in the $\mathrm{NAC}+\mathrm{CHO}$ group than in the NAC group or non-NAC group $(P<0.05$; Table 4$)$.

3.5. CD8\% in Peripheral Venous Blood. As detailed in Table 5, there were no significant differences between groups in $\mathrm{CD}^{+}$ (the percentage of cells that were cytotoxic T cells) on the day 
TABLE 6: $\mathrm{CD}^{+} / \mathrm{CD}^{+}$ratio in peripheral venous blood.

\begin{tabular}{lcccc}
\hline Time point & Non-NAC group & NAC group & NAC+CHO group & $F^{2}$ \\
\hline Day after first admission & $1.75 \pm 0.54$ & $2.09 \pm 1.05$ & $0.97 \pm 0.99$ & 0.998 \\
Before surgery & $1.73 \pm 0.44$ & $2.75 \pm 0.80^{\mathrm{a}, 1}$ & $2.78 \pm 1.39^{\mathrm{a}, 1}$ & 19.585 \\
After tracheal tube removal & $1.11 \pm 0.16^{1,2}$ & $2.10 \pm 0.70^{\mathrm{a}, 2}$ & $2.21 \pm 1.04^{\mathrm{a}}$ & 0.375 \\
Day after surgery & $0.91 \pm 0.17^{1,2}$ & $2.09 \pm 0.40^{\mathrm{a}, 2}$ & $2.42 \pm 0.73^{\mathrm{a}, \mathrm{b}}$ & 20.768 \\
$F^{1}$ & 38.657 & 4.501 & 2.436 & 0.459 \\
$P^{1}$ & 0.000 & 0.013 & 0.090 \\
${ }^{1} P<0.05$ vs. day after the first admission; ${ }^{2} P<0.05$ vs. before surgery; ${ }^{3} P<0.05$ vs. after tracheal tube removal; ${ }^{\mathrm{a}} P<0.05$ vs. the non-NAC group; ${ }^{\mathrm{b}} P<0.05$ vs. \\
the NAC group.
\end{tabular}

after the first admission, although CD8\% was higher in the non-NAC group than in the other two groups just before surgery due to a fall in $\mathrm{CD} 8 \%$ in the NAC and $\mathrm{NAC}+\mathrm{CHO}$ groups $(P<0.05)$. Postoperatively, there were significant increases in $\mathrm{CD} 8 \%$ in the non-NAC and NAC groups $(P<0.05)$, whereas no such increase occurred in the NAC $+\mathrm{CHO}$ group (Table 5).

3.6. $\mathrm{CD}^{+} / \mathrm{CD}^{+}$Ratio in Peripheral Venous Blood. Table 6 demonstrates that there were no significant differences between the three groups in the $\mathrm{CD} 4^{+} / \mathrm{CD}^{+}$ratio on the day after the first admission. However, the $\mathrm{CD} 4^{+} / \mathrm{CD}^{+}$ratio was significantly lower in the non-NAC group than in the other two groups just before surgery $(P<0.05)$, reflecting the decline in $\mathrm{CD} 8 \%$ in the $\mathrm{NAC}$ and $\mathrm{NAC}+\mathrm{CHO}$ groups at this time point (see above). Compared with the value just before surgery, the non-NAC and NAC groups exhibited significant decreases in the $\mathrm{CD} 4^{+} / \mathrm{CD}^{+}$ratio $(P<0.05)$, whereas little or no change was observed in the $\mathrm{NAC}+\mathrm{CHO}$ group (Table 6).

\section{Discussion}

A notable finding of this study was that women with cervical cancer exhibited decreases in NK\%, CD3\%, and CD4\% and an increase in $\mathrm{CD} 8 \%$ after $\mathrm{RH}$ with PLND. However, the changes in $\mathrm{NK} \%, \mathrm{CD} 3 \%, \mathrm{CD} 4 \%$, and $\mathrm{CD} 8 \%$ were less pronounced in the NAC-CHO group than in the NAC and non-NAC groups. These data suggest that preoperative oral carbohydrate might help to reduce disturbances in immune cell numbers in patients treated surgically for cervical cancer.

Antitumor immunity mainly relies on the cellular immune system, including T cell subsets and NK cells [29]. $\mathrm{T}_{\mathrm{H}} 1$ helper cells exert antitumor effects via recruitment of CD8 ${ }^{+}$cytotoxic T cells, NK cells, and macrophages that kill cancer cells. $\mathrm{T}_{\mathrm{H}} 2$ helper cells have antitumor actions through the recruitment of eosinophils [29]. $\mathrm{CD}^{+}$cytotoxic $\mathrm{T}$ cells and NK cells can target and kill tumor cells [29]. By contrast, $\mathrm{CD}^{+}$regulatory T cells suppress antitumor immunity [29]. Cancer cells express various cytokines that inhibit lymphocyte differentiation/proliferation, reduce $\mathrm{CD}^{+}$cell levels, and increase $\mathrm{CD} 8 \%$, which further inhibits $\mathrm{CD}^{+}{ }^{+} \mathrm{T}$ lymphocytes in a vicious circle [30]. This suppression of normal immune responses facilitates tumor growth and proliferation [12]. With regard to cervical cancer, previous research has revealed changes in $\mathrm{T}$ cell subsets and NK cell populations as well as inhibition of $\mathrm{NK}$ cell activity by $\mathrm{CD} 4^{+}$regulatory $\mathrm{T}$ cells [13-15]. Thus, it is important that disturbances in immune function are minimized during the treatment of cervical cancer.

NAC can decrease cervical tumor size, improve surgical feasibility, improve progression-free survival and overall survival, and reduce the requirement for postoperative adjuvant therapy [6-11, 31]. Paclitaxel and cisplatin selectively kill tumor cells and may improve immune status in patients with cancer $[32,33]$. Some studies have observed no effects of NAC on lymphocyte numbers in patients with cervical cancer $[17,18]$. However, chemotherapy has been reported to inhibit NK cell activity [19]. In the present study, $\mathrm{NK} \%$, CD3\%, and CD4\% were not significantly different between the three groups at both preoperative time points. This suggests that the effects of NAC on NK\%, $\mathrm{CD} 3 \%$, and $\mathrm{CD} 4 \%$ were either negligible or short-lasting (i.e., <4 weeks). However, preoperative CD8\% was lower in the NAC groups than in the non-NAC group. This suggests a possible effect of NAC on $\mathrm{CD}^{+}$lymphocyte numbers, as reported previously in an animal study [34]. If so, NAC might be a "double-edged sword" in that it not only targets tumor cells but also reduces cytotoxic $\mathrm{T}$ cell function and thus antitumor immunity.

Surgery can cause an immunosuppressive effect in the early postoperative stage due to surgical stress and trauma, effects of general anesthetic agents, intraoperative hypothermia, intraoperative blood loss and transfusion, and hyperglycemia due to postoperative insulin resistance [35-37]. Major surgery can impair cell-mediated immunity in the short term, with decreases in tumor necrosis factor-alpha, interleukin2, interferon-gamma, and lymphoproliferation [38]. Consistent with our findings, a previous investigation reported that $\mathrm{RH}$ for cervical cancer reduced $\mathrm{CD} 4^{+} \mathrm{T}$ cell and $\mathrm{NK}$ cell numbers [16]. It is possible that immune dysfunction after surgical treatment of cervical cancer may impair antitumor immunity.

Preoperative oral carbohydrate can attenuate postoperative reductions in T cell and NK cell numbers [23]. We also found that a carbohydrate drink given 2 hours before surgery limited the postoperative changes in NK\%, CD3\%, and CD $4 \%$. Preoperative oral carbohydrate may have several benefits in patients undergoing major surgery, including a reduction in postoperative insulin resistance and blood glucose levels [21-28]. In healthy people, hyperglycemia is associated with decreased lymphocyte counts [39]. Furthermore, severe 
perioperative hyperglycemia can suppress $\mathrm{T}$ cell activation, basophil count, and monocyte function [40]. We speculate that the beneficial effects of preoperative oral carbohydrate on postoperative $\mathrm{NK} \%, \mathrm{CD} 3 \%$, and CD4\% were due to suppression of perioperative insulin resistance and hyperglycemia. However, additional studies are needed to establish the validity of this hypothesis.

The benefit of oral carbohydrates on tumor immunity before surgery is mainly related to increased blood glucose levels. The effect of blood glucose levels on the immune system is also reflected in the production of lymphocytes. Indeed, in healthy subjects, in the state of hyperglycemia, the lymphocytes and their subpopulations are decreased, while the abnormalities decrease when the blood glucose decrease [41]. At the same time, hyperglycemia is closely related to the increase of cytotoxic $\mathrm{T}$ cells and the decrease of immune $\mathrm{T}$ cells. The negative regulation of cell activity during hyperglycemia is due to the decrease in CD4+/CD8+\% and the enhanced expression of HLA-DR antigens [42], thus ensuring the stability of serum environmental glucose levels and possibly can avoid the interference with normal cells to execute normal activities, thereby ensuring their selfregulating function, shortening the inflammatory processes, and playing a positive effect on the body's immunity. Nevertheless, the exact mechanisms remain to be determined.

This study has some limitations. This was a single-center study. The different tumor types and chemotherapy regimens introduced some biases in the analyses. Neither the patients nor investigators were blinded to the intervention, which may have introduced bias. In addition, the generalizability of the findings is unknown. The sample size was small, so the study may have been underpowered to detect some real differences between groups. The non-NAC group enrolled patients with a different disease stage to those in the NAC groups, which may have introduced bias. The patients were followed up for only 1 day after surgery, so longer-term effects were not determined. We did not perform a safety analysis.

\section{Conclusion}

Preoperative oral carbohydrate treatment may help to attenuate the changes in NK and T cell populations after treatment of cervical cancer by NAC and surgery. However, large-scale, multicenter, randomized controlled trials are required to validate our findings and establish whether preoperative oral carbohydrate might reduce disease recurrence or improve survival rate.

\section{Data Availability}

The datasets used and/or analyzed during the current study are available from the corresponding author on reasonable request.

\section{Ethical Approval}

This study was approved by the Institutional Medical Ethics Committee of Fujian Cancer Hospital \& Fujian Medical University Cancer Hospital (KT2018-003-016513).

\section{Consent}

Written informed consent was obtained from all participants. The trial was registered at clinicaltrials.gov (NCT03872635; PI: $\mathrm{H}$ Ping; date of registration: 03/10/2019).

\section{Conflicts of Interest}

The authors declare that they have no competing interests.

\section{Authors' Contributions}

FQZ generated the experimental hypothesis, designed the study, analyzed the experimental data, and wrote the manuscript. MXY participated in the study design and helped revise the manuscript. MXY and ZPL contributed to the data collection. WHC participated in the study design, analyses, and interpretation of data and critically reviewed the manuscript. All authors read and approved the final manuscript.

\section{Acknowledgments}

This study was supported by the Science and Technology Program of Fujian Province, China (No. 2018Y2003). The authors thank the patients and staff who participated in this study.

\section{References}

[1] L. A. Torre, F. Bray, R. L. Siegel, J. Ferlay, J. Lortet-Tieulent, and A. Jemal, "Global cancer statistics, 2012," CA: A Cancer Journal for Clinicians, vol. 65, no. 2, pp. 87-108, 2015.

[2] W. Chen, R. Zheng, P. D. Baade et al., "Cancer statistics in China, 2015," CA: A Cancer Journal for Clinicians, vol. 66, no. 2, pp. 115-132, 2016.

[3] S. P. Somashekhar and K. R. Ashwin, "Management of early stage cervical cancer," Reviews on Recent Clinical Trials, vol. 10, no. 4, pp. 302-308, 2015.

[4] S. R. Carneiro, M. A. Fagundes, P. J. O. do Rosário, L. M. T. Neves, G. S. Souza, and M. C. N. Pinheiro, "Five-year survival and associated factors in women treated for cervical cancer at a reference hospital in the Brazilian Amazon," PloS One, vol. 12, no. 11, article e0187579, 2017.

[5] W. J. Koh, N. R. Abu-Rustum, S. Bean et al., "Cervical cancer, version 3.2019, NCCN clinical practice guidelines in oncology," Journal of the National Comprehensive Cancer Network: JNCCN, vol. 17, no. 1, pp. 64-84, 2019.

[6] L. Kumar, R. Pramanik, S. Kumar, N. Bhatla, and S. Malik, "Neoadjuvant chemotherapy in gynaecological cancers implications for staging," Best Practice \& Research Clinical Obstetrics \& Gynaecology, vol. 29, no. 6, pp. 790-801, 2015.

[7] P. Benedetti Panici, I. Palaia, C. Marchetti et al., "Dose-dense neoadjuvant chemotherapy plus radical surgery in locally advanced cervical cancer: a phase II study," Oncology, vol. 89, no. 2, pp. 103-110, 2015.

[8] A. S. Mousavia, S. Vahidi, M. Karimi-Zarchi, M. ModarressGilania, and F. Ghaemmaghamia, "Response to neoadjuvant chemotherapy with paclitaxel and cisplatin in locally advanced cervical cancer," European Journal of Gynaecological Oncology, vol. 34, no. 6, pp. 527-531, 2013.

[9] R. Zhang, B. Li, P. Bai et al., "Neoadjuvant chemotherapy with paclitaxel and cisplantin or carboplatin for patients with 
locally advanced uterine cervical cancer," Zhonghua zhong liu za zhi [Chinese Journal of Oncology], vol. 33, no. 8, pp. 616620, 2011.

[10] N. Prueksaritanond, P. Chaisarn, and M. Yanaranop, “The efficacy of neoadjuvant paclitaxel-carboplatin chemotherapy followed by radical hysterectomy compared to radical hysterectomy alone in bulky stage IB2-IIA cervical cancer," Journal of the Medical Association of Thailand, vol. 95, Suppl 3, pp. S55-S61, 2012.

[11] L. Rydzewska, J. Tierney, C. L. Vale, and P. R. Symonds, "Neoadjuvant chemotherapy plus surgery versus surgery for cervical cancer," The Cochrane Database of Systematic Reviews, no. 1, article CD007406, 2008.

[12] M. R. Rutkowski, N. Svoronos, A. Perales-Puchalt, and J. R. Conejo-Garcia, "The tumor macroenvironment: cancerpromoting networks beyond tumor beds," Advances in Cancer Research, vol. 128, pp. 235-262, 2015.

[13] O. V. Kurmyshkina, P. I. Kovchur, L. V. Schegoleva, and T. O. Volkova, "T- and NK-cell populations with regulatory phenotype and markers of apoptosis in circulating lymphocytes of patients with CIN3 or microcarcinoma of the cervix: evidence for potential mechanisms of immune suppression," Infectious Agents and Cancer, vol. 12, no. 1, 2017.

[14] W. C. Chang, C. H. Li, L. H. Chu, P. S. Huang, B. C. Sheu, and S. C. Huang, "Regulatory T cells suppress natural killer cell immunity in patients with human cervical carcinoma," International Journal of Gynecological Cancer: Official Journal of the International Gynecological Cancer Society, vol. 26, no. 1, pp. 156-162, 2016.

[15] M. C. Bell, R. P. Edwards, E. E. Partridge et al., "CD8+ T lymphocytes are recruited to neoplastic cervix," Journal of Clinical Immunology, vol. 15, no. 3, pp. 130-136, 1995.

[16] S. Liu, X. Gu, L. Zhu et al., "Effects of propofol and sevoflurane on perioperative immune response in patients undergoing laparoscopic radical hysterectomy for cervical cancer," Medicine, vol. 95, no. 49, article e5479, 2016.

[17] H. R. Marana, J. M. Andrade, and J. S. Silva, "Natural killer cells and interleukin-12 in patients with advanced cervical cancer under neoadjuvant chemotherapy," Brazilian Journal of Medical and Biological Research $=$ Revista brasileira de pesquisas medicas e biologicas, vol. 29, no. 4, pp. 473-477, 1996.

[18] H. R. Cosiski Marana, J. Santana da Silva, and J. Moreira de Andrade, "NK cell activity in the presence of IL-12 is a prognostic assay to neoadjuvant chemotherapy in cervical cancer," Gynecologic Oncology, vol. 78, no. 3, pp. 318-323, 2000.

[19] G. G. Garzetti, A. Ciavattini, M. Provinciali, H. Valensise, C. Romanini, and N. Fabris, "Influence of neoadjuvant polychemotherapy on natural killer cell activity in patients with locally advanced cervical squamous carcinoma," Gynecologic Oncology, vol. 52, no. 1, pp. 39-43, 1994.

[20] D. A. Fraser, J. Thoen, J. E. Reseland, O. Forre, and J. KjeldsenKragh, "Decreased CD4+ lymphocyte activation and increased interleukin-4 production in peripheral blood of rheumatoid arthritis patients after acute starvation," Clinical Rheumatology, vol. 18, no. 5, pp. 394-401, 1999.

[21] J. Nygren, A. Thorell, H. Jacobsson et al., "Preoperative gastric emptying. Effects of anxiety and oral carbohydrate administration," Annals of Surgery, vol. 222, no. 6, pp. 728-734, 1995.

[22] M. D. Smith, J. McCall, L. Plank et al., "Preoperative carbohydrate treatment for enhancing recovery after elective surgery," The Cochrane Database of Systematic Reviews, no. 8, article CD009161, 2014.
[23] J. Nygren, A. Thorell, and O. Ljungqvist, "Preoperative oral carbohydrate therapy," Current Opinion in Anaesthesiology, vol. 28, no. 3, pp. 364-369, 2015.

[24] Y. Oyama, H. Iwasaka, K. Shiihara et al., "Effects of preoperative oral carbohydrates and trace elements on perioperative nutritional status in elective surgery patients," Middle East Journal of Anaesthesiology, vol. 21, no. 3, pp. 375-383, 2011.

[25] M. Kaška, T.'. Grosmanová, E. Havel et al., “The impact and safety of preoperative oral or intravenous carbohydrate administration versus fasting in colorectal surgery-a randomized controlled trial," Wiener Klinische Wochenschrift, vol. 122, no. 1-2, pp. 23-30, 2010.

[26] E. Cakar, E. Yilmaz, E. Cakar, and H. Baydur, "The effect of preoperative oral carbohydrate solution intake on patient comfort: a randomized controlled study," Journal of Perianesthesia Nursing: Official Journal of the American Society of Peri Anesthesia Nurses, vol. 32, no. 6, pp. 589-599, 2017.

[27] L. Gianotti, R. Biffi, M. Sandini et al., "Preoperative oral carbohydrate load versus placebo in major elective abdominal surgery (PROCY): a randomized, placebo-controlled, Multicenter, Phase III Trial," Annals of Surgery, vol. 267, no. 4, pp. 623-630, 2018.

[28] D. K. Bilku, A. R. Dennison, T. C. Hall, M. S. Metcalfe, and G. Garcea, "Role of preoperative carbohydrate loading: a systematic review," Annals of the Royal College of Surgeons of England, vol. 96, no. 1, pp. 15-22, 2014.

[29] H. J. Kim and H. Cantor, "CD4 T-cell subsets and tumor immunity: the helpful and the not-so-helpful," Cancer Immunology Research, vol. 2, no. 2, pp. 91-98, 2014.

[30] M. Pace, J. Williams, A. Kurioka et al., "Histone deacetylase inhibitors enhance CD4 T cell susceptibility to NK cell killing but reduce NK cell function," PLoS Pathogens, vol. 12, no. 8, article e1005782, 2016.

[31] A. Maneo, S. Chiari, C. Bonazzi, and C. Mangioni, "Neoadjuvant chemotherapy and conservative surgery for stage IB1 cervical cancer," Gynecologic Oncology, vol. 111, no. 3, pp. 438443, 2008.

[32] R. Liu, J. Zhang, C. He, Q. Jiang, J. Liu, and R. Fan, "Impact of the radiotherapy combined with cisplatin plus paclitaxel chemotherapy on the immunologic functions in the patients with esophageal cancer," Pakistan Journal of Pharmaceutical Sciences, vol. 29, 4 Suppl, pp. 1387-1390, 2016.

[33] D. Krantz, C. A. Hartana, M. E. Winerdal et al., "Neoadjuvant Chemotherapy Reinforces Antitumour $\mathrm{T}$ cell Response in Urothelial Urinary Bladder Cancer," European Urology, vol. 74, no. 6, pp. 688-692, 2018.

[34] Y. Chu, L. X. Wang, G. Yang et al., "Efficacy of GM-CSFproducing tumor vaccine after docetaxel chemotherapy in mice bearing established Lewis lung carcinoma," Journal of Immunotherapy, vol. 29, no. 4, pp. 367-380, 2006.

[35] Y. Chen, M. Liang, Y. Zhu, and D. Zhou, “The effect of propofol and sevoflurane on the perioperative immunity in patients under laparoscopic radical resection of colorectal cancer," Zhonghua Yi Xue Za Zhi, vol. 95, no. 42, pp. 3440-3444, 2015.

[36] H. D. T. Torrance, E. R. Longbottom, M. E. Vivian et al., "Postoperative immune suppression is mediated via reversible, interleukin-10 dependent pathways in circulating monocytes following major abdominal surgery," PloS One, vol. 13, no. 9, article e0203795, 2018.

[37] F. Hildebrand, M. van Griensven, P. Giannoudis et al., "Impact of hypothermia on the immunologic response after trauma 
and elective surgery," Surgical Technology International, vol. 14, pp. 41-50, 2005.

[38] G. Amodeo, D. Bugada, S. Franchi et al., "Immune function after major surgical interventions: the effect of postoperative pain treatment," Journal of Pain Research, vol. Volume 11, pp. 1297-1305, 2018.

[39] R. von Kanel, P. J. Mills, and J. E. Dimsdale, "Short-term hyperglycemia induces lymphopenia and lymphocyte subset redistribution," Life Sciences, vol. 69, no. 3, pp. 255-262, 2001.

[40] G. Lachmann, C. von Haefen, T. Wollersheim, and C. Spies, "Severe perioperative hyperglycemia attenuates postoperative monocytic function, basophil count and T cell activation," Minerva Anestesiologica, vol. 83, no. 9, pp. 921-929, 2017.

[41] J. Steiner, H. G. Bernstein, K. Schiltz et al., "Immune system and glucose metabolism interaction in schizophrenia: A chickenegg dilemma," Progress in Neuro-Psychopharmacology \& Biological Psychiatry, vol. 48, pp. 287-294, 2014.

[42] M. Madani, K. Alizadeh, S. P. Ghazaee et al., "Elective percutaneous coronary intervention: the relationship between preprocedural blood glucose levels and periprocedural myocardial injury," Texas Heart Institute Journal, vol. 40, no. 4, pp. 410417, 2013. 